Revista Brasileira de Agricultura Irrigada v.8, no. 6, p.466 - 475, 2014

ISSN 1982-7679 (On-line)

Fortaleza, CE, INOVAGRI - http://www.inovagri.org.br

DOI: $10.7127 /$ rbai.v8n600260

Protocolo 260.14 - 24/07/2014 Aprovado em 22/09/2014

\title{
CRESCIMENTO INICIAL DO AMENDOINZEIRO IRRIGADO COM ÁGUAS SALINAS EM DIFERENTES SUBSTRATOS
}

Fellype Rodrigo Barroso Costa ${ }^{1}$, Krishna Ribeiro Gomes ${ }^{1}$, Geocleber Gomes de Sousa ${ }^{2}$, Benito Moreira de Azevedo ${ }^{3}$, Fabio Josias Farias Monteiro ${ }^{4}$, Thales Vinícius de Araújo Viana $^{3}$

\section{RESUMO}

Objetivou-se com esse trabalho avaliar a interação entre o estresse salino da água de irrigação e substratos com fertilizantes orgânicos no crescimento e na biomassa de plantas de amendoim. $\mathrm{O}$ trabalho foi conduzido na área experimental da Estação Agrometeorológica, UFC, em Fortaleza, no período de setembro a novembro de 2013. O delineamento experimental empregado foi inteiramente casualizado, num esquema fatorial $4 \times 2$, referente aos fertilizantes orgânicos aplicado no substrato $(\mathrm{T} 1=$ areia + arisco; T2 = areia + arisco + biofertilizante bovino de fermentação aeróbia; T3= areia + arisco + biofertilizante bovino de fermentação anaeróbia e $\mathrm{T} 4=$ areia + arisco + biofertilizante de caranguejo), com dois níveis de salinidade da água $\left(1,0\right.$ e 4,0 dS m $\left.\mathrm{d}^{-1}\right) \mathrm{e}$ 5 repetições. $\mathrm{O}$ ensaio foi conduzido em vasos de $10 \mathrm{~L}$, sendo uma planta por vaso. Aos 45 dias após a semeadura foram analisadas as seguintes variáveis: número de folhas, altura de plantas, área foliar, matéria seca da parte aérea, matéria seca da raiz e matéria seca total. O biofertilizante de caranguejo apresentou-se eficiente na melhoria da produção do amendoim em água salina.O substrato com biofertilizante bovino de fermentação aeróbia foi mais eficiente sob a área foliar e o número de folhas sob irrigação com água de baixa e alta salinidade. O substrato contendo biofertilizante bovino de fermentação anaeróbia exerce efeito atenuador para a altura de plantas irrigadas com água de baixa e alta salinidade.

Palavras-chave: Arachis hypogaea L.. estresse salino. fertilizante orgânico. oleaginosa.

\footnotetext{
${ }^{1}$ Mestre em Engenharia Agrícola, Departamento de Engenharia Agrícola, Universidade Federal do Ceará, Fortaleza, Brasil, Campus do Pici, CCA/UFC, Av. Mister Hull, 2977, CEP 60455-760, Fortaleza, CE, email: fellyperodrigo@yahoo.com.br

2 Doutor em Engenharia Agrícola, Bolsista de PNPD/CAPES/UFC, Departamento de Engenharia Agrícola, Universidade Federal do Ceará, Fortaleza, Brasil, Campus do Pici, CCA/UFC, Fortaleza, CE, email: sousasolosgeo@hotmail.com

${ }_{3}^{3}$ Professor do Departamento de Engenharia Agrícola, Universidade Federal do Ceará, Fortaleza, Brasil, Campus do Pici,CCA/UFC, Fortaleza, CE, e-mail: benitoazevedo@ hotmail.com; thales@ufc.br

${ }^{4}$ Doutorando Engenharia Agrícola, Departamento de Engenharia Agrícola, Universidade Federal do Ceará, Fortaleza, Brasil, Campus do Pici, CCA/UFC, Fortaleza, CE, e-mail: fjfm.agro@gmail.com
} 


\title{
INITIAL GROWTH OF PEANUT IRRIGATED WITH SALINE WATER ON DIFFERENT SUBSTRATES
}

\begin{abstract}
The objective of this study was to evaluate the effect of salinity of irrigation water in the biomass of peanut plants in substrates with organic fertilizers. The work was conducted in the experimental area of the weather station, UFC, Fortaleza, in the period September-November 2013. Experimental design was completely randomized in a $4 \times 2$ factorial design, referring to the organic fertilizers applied on the substrate $(\mathrm{T} 1=$ sand + aloof, $\mathrm{T} 2=$ sand + aloof + bovine biofertilizer with aerobic fermentation, $\mathrm{T} 3=$ sand + aloof + bovine biofertilizer with anaerobic fermentation and T4 $=$ sand + aloof + crab biofertilizer), with two levels of salinity $\left(1.0\right.$ and $\left.40 \mathrm{dS} \mathrm{m}^{-1}\right)$ and 5 replicates. The trial was conducted in 10-liter pots, one plant per pot. 45 days after sowing, the following variables were analyzed: number of leaves, plant height, leaf area, shoot dry matter, root dry matter and total dry matter. Crab biofertilizer presented itself efficient in improving the production of peanut in saline water. The substrate with bovine biofertilizer with aerobic fermentation was more efficient at the leaf area and the number of leaves in irrigation with low and high salinity water. The substrate containing bovine biofertilizer with anaerobic fermentation exerts mitigating effect to the height of plants irrigated with low water and high salinity.
\end{abstract}

Keywords: Arachis hypogaea L., organic fertilizer, rape, salt stress.

\section{INTRODUÇÃO}

O amendoim (Arachis hypogaea L.) é uma oleaginosa economicamente muito importante em âmbito mundial, pertencente à família Fabaceae, tendo uma produção de 1,92 milhões de toneladas (USDA, 2013). No Brasil, a produção na safra 2013/2014 poderá chegar à 316,7 mil toneladas. Essa produção se concentra na região Sudeste, especialmente no estado de São Paulo, que conta com uma participação em torno de $96 \%$ do total nacional (CONAB, 2013).

A salinidade dos solos é um dos principais fatores ambientais que limitam a produtividade agrícola devido aos seus efeitos no crescimento e desenvolvimento vegetal, os quais podem ser de natureza iônica e, ou osmótica, uma vez que a condutividade elétrica dos solos salinos é igual ou superior a $4,0 \mathrm{dS} \mathrm{m} \mathrm{m}^{-1}$, que corresponde a uma concentração aproximada de $40 \mathrm{nM}$ de $\mathrm{NaCl}$ e uma pressão osmótica de 0,2 MPa (MUNNS e TESTER, 2008).

No Nordeste, a salinização dos solos é atribuída ao fato de que a evapotranspiração potencial da região, geralmente, é menor do que a precipitação pluvial (SILVA \& AMARAL, 2007). Esse fator, associado ao manejo inadequado da água e do solo, tem afetado a produtividade das culturas produzidas na região. $\mathrm{O}$ aumento do teor salino na água de irrigação ou do solo diminui o potencial osmótico da solução, reduzindo a disponibilidade de água e nutrientes para as plantas (SOUSA et al., 2010).

O biofertilizante é um adubo orgânico líquido produzido em meio aeróbico ou anaeróbico a partir de uma mistura de material orgânico fresco e água (PENTEADO, 2007). Apresenta em sua composição nutrientes essenciais ao desenvolvimento das culturas, que possibilitam a redução da utilização de produtos 
químicos, gerando economia de insumos e melhora os atributos físicos e biológicos do solo. Além disso, o biofertilizante bovino vem sendo utilizado como uma das estratégias de manejo que possibilitam a exploração de áreas irrigadas com água salina na agricultura (SILVA et al., 2011). Nesse trabalho, esses autores relatam que mesmo sob estresse salino, o uso desse insumo orgânico proporcionou maiores valores de fotossíntese, condutância estomática e transpiração em plantas de feijãode-corda cultivadas em vasos, 45 dias após a semeadura.

Segundo Sousa et al., (2012a) esse insumo orgânico atenuou o estresse salino em solo cultivado com a cultura do amendoim aos 45 dias após a semeadura. Estudos sobre como amenizar os efeitos da salinidade sobre as plantas são importantes, ou seja, o excesso de sais pode comprometer as funções fisiológicas e bioquímicas das plantas, causando estresse osmótico, o que resulta em alterações na absorção e utilização de nutrientes essenciais além do acúmulo de íons tóxicos (SOUSA et al., 2010; CALVET et al., 2010).

Utilizar esses solos implica na redução do crescimento, do desenvolvimento e dos teores de macronutrientes catiônicos das plantas (GARCIA et al., 2007; BAGHALIAN et al., 2008). A partir do exposto, objetivou-se com esse trabalho avaliar a interação entre o estresse salino da água de irrigação e substratos com fertilizantes orgânicos no crescimento e na biomassa de plantas de amendoim.

\section{MATERIAL E MÉTODOS}

O trabalho foi conduzido a pleno sol em uma área experimental da Estação Agrometeorológica, UFC, em Fortaleza, Ceará, $\left(3^{\circ} 45^{\prime} \mathrm{S} ; 38^{\circ} 33^{\prime} \mathrm{W}\right.$ e altitude de $\left.19 \mathrm{~m}\right)$, no período de setembro a novembro de 2013. Segundo a classificação de Köppen, a área do experimento está localizada numa região de clima Aw', caracterizado como tropical chuvoso, muito quente, com chuvas predominantes nas estações do verão e do outono.

O delineamento experimental utilizado foi o inteiramente casualizado, em arranjo fatorial 4 x 2, com cinco repetições. Os tratamentos foram constituídos de quatro fertilizantes orgânicos aplicados no substrato $(\mathrm{T} 1=$ areia + arisco; $\mathrm{T} 2=$ areia + arisco + biofertilizante bovino de fermentação aeróbia; T3= areia + arisco + biofertilizante bovino de fermentação anaeróbia e $\mathrm{T} 4=$ areia + arisco + biofertilizante de caranguejo), com duas condutividades elétricas

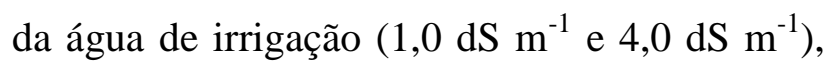
aplicadas aos vasos. O biofertilizante foi diluído em água na razão de 1:1, aplicado de uma única vez, em volume equivalente a $10 \%(1,0 \mathrm{~L}$ planta $^{-1}$ ) do volume do substrato. Foram utilizadas sementes de amendoim BR-1, as quais foram plantadas em vasos plásticos com capacidade de 10 litros. Após o estabelecimento das plântulas, aos 8 dias após a semeadura (DAS), fez-se o desbaste deixando-se uma planta por vaso.

O biofertilizante bovino de fermentação aeróbia foi preparado a partir de uma mistura de esterco bovino e água na proporção 1:1 com base em volume. Nesse sistema a mistura foi armazenada em uma caixa d'água com capacidade para 1000 litros, e permaneceu no tanque por um período de mínimo de 30 dias sofrendo fermentação aeróbia. Semanalmente era realizada a homogeneização desse sistema 
de forma manual. O biofertilizante bovino de fermentação anaeróbia foi preparado a partir de uma mistura de partes iguais de esterco fresco bovino e água não salina $\left(\mathrm{CEa}=0,8 \mathrm{dS} \mathrm{m}^{-1}\right) \mathrm{sob}$ fermentação anaeróbia, durante 30 dias, em recipiente plástico. Para se obter o sistema anaeróbio, a mistura foi colocada em uma bombona plástica de $240 \mathrm{~L}$ deixando-se um espaço vazio de 15 a $20 \mathrm{~cm}$ na parte superior e fechada hermeticamente. Na tampa foi adaptada uma mangueira com a outra extremidade mergulhada num recipiente com água na altura de $20 \mathrm{~cm}$, para a saída de gases (PENTEADO, 2007). O biofertilizante de caranguejo foi produzido de forma semelhante ao biofertilizante aeróbio, sendo que ele recebeu em sua composição pedaços de rapadura $(2 \mathrm{~kg})$ em forma de melaço.

$\mathrm{Na}$ preparação da água salina foram utilizados os sais de $\mathrm{NaCl}, \mathrm{CaCl}_{2} \cdot 2 \mathrm{H}_{2} \mathrm{O}$ e $\mathrm{MgCl}_{2} \cdot 6 \mathrm{H}_{2} \mathrm{O}$, na proporção de 7:2:1 (MEDEIROS, 1992). A irrigação com as fontes de água de diferentes salinidades foi iniciada após o desbaste (8 DAS) - dias após o semeio e a quantidade de água aplicada diariamente às plantas foi calculada de acordo com o princípio do lisímetro de drenagem (BERNARDO et al., 2008), mantendo-se o solo na capacidade de campo.

Aos 45 dias após a semeadura foram analisadas as seguintes variáveis: número de folhas, altura de plantas, área foliar, matéria seca da parte aérea, matéria seca da raiz e matéria seca total. A altura das plantas foi medida utilizando-se uma régua milimetrada e a área foliar foi determinada utilizando-se um medidor de área foliar (LI - 3100, Area Meter, Li - Cor., In. Lincoln, Nebraska, USA) colocando-se a parte aérea (folha) no medidor para realização da leitura.

Os dados obtidos foram submetidos a análises estatísticas com o auxílio do software ASSISTAT versão 7,6 beta (2011).

\section{RESULTADOS E DISCUSSÃO}

O substrato de biofertilizante de caranguejo proporcionou o maior valor de massa seca da parte aérea (folha), diferindo estatisticamente dos demais substratos analisados (Tabela 1). Esse resultado corrobora com o encontrado por Sousa et al. (2013), em estudos realizados com feijão-de-corda em solos com biofertilizantes e águas salinas em Fortaleza, Ceará.

Tabela 1. Valores médios de matéria seca da parte aérea de amendoim cultivado em diferentes substratos.

\begin{tabular}{cccc}
\hline \multicolumn{4}{c}{ MSPA } \\
\hline \multicolumn{4}{c}{ Substratos } \\
\hline Areia+arisco $^{1}$ & Caranguejo & Anaeróbio & Aeróbio \\
$1,58 \mathrm{c}$ & $7,46 \mathrm{a}$ & $1,45 \mathrm{c}$ & $4,35 \mathrm{~b}$ \\
\hline $\mathrm{MG}$ & 3,71 & $\mathrm{CV}$ & 30,35 \\
DMS & 2,47 & & \\
\hline
\end{tabular}

I=Testemunha; Médias seguidas da mesma letra minúscula na linha não diferem estatisticamente entre si (Tukey 0,05); DMS=diferença mínima significativa; $\mathrm{CV}=$ coeficiente de variação; $\mathrm{MG}=$ média geral.

O maior valor apresentado por este substrato contradiz o resultado que foi apresentado por Sousa et al. (2012a), que, estudando a cultura do amendoim sob diferentes níveis de água salina em substratos com biofertilizante, encontrou valores maiores de 
matéria seca da parte aérea no substrato com fermentação anaeróbia.

Já em relação à matéria seca da raiz, os valores diferiram em relação a substrato e condutividade elétrica da água de irrigação (Tabela 2). O substrato do caranguejo apresentou as melhores médias de matéria seca da raiz nas duas condutividades elétricas da água de irrigação, sendo o único a ter incremento significativo na produção de matéria seca com o aumento dos valores de CE. Esse valor se mostra contrário ao apresentado por Santos et al. (2013), que, estudando o biofertilizante de caranguejo aplicado na cultura do milho, apresentou resultados em que a produção de matéria seca da raiz decrescia com o aumento da condutividade elétrica da água. Os outros substratos estudados não apresentaram diferença significativa na produção de matéria seca de raiz em relação ao aumento da CE.

Tabela 2. Valores médios de matéria seca da raiz de amendoim cultivado em diferentes substratos sob diferentes níveis de salinidade.

\begin{tabular}{|c|c|c|c|c|}
\hline \multirow[t]{3}{*}{$\begin{array}{l}\text { Água de } \\
\text { irrigação }\end{array}$} & \multicolumn{4}{|c|}{ MSR } \\
\hline & \multicolumn{4}{|c|}{ Substratos } \\
\hline & Areia+arisco & Caranguejo & Anaeróbio & Aeróbio \\
\hline $0,8 \mathrm{dS} \mathrm{m}^{-1}$ & $0,3 \mathrm{bA}$ & $0,96 \mathrm{aB}$ & $0,16 \mathrm{bA}$ & $0,4 \mathrm{bA}$ \\
\hline \multirow[t]{3}{*}{$4 \mathrm{dS} \mathrm{m}^{-1}$} & $0,13 \mathrm{cA}$ & $1,26 \mathrm{aA}$ & $0,2 \mathrm{bcA}$ & $0,46 \mathrm{bA}$ \\
\hline & MG & 0,48 & $\mathrm{CV}$ & 24,77 \\
\hline & DMS coluna & 0,28 & DMS linha & 0,2 \\
\hline \multicolumn{5}{|c|}{$\begin{array}{l}\text { Médias seguidas da mesma letra minúscula não diferem } \\
\text { estatisticamente entre si em relação a substrato. Médias } \\
\text { seguidas da mesma letra maiúscula não diferem } \\
\text { estatisticamente entre si em relação a CE (Tukey } 0,05 \text { ); } \\
\text { DMS=diferença mínima significativa; } C V=\text { coeficiente de } \\
\text { variação; } M G=\text { média geral. }\end{array}$} \\
\hline
\end{tabular}

Os valores médios de matéria seca total produzida pelo amendoim nos diferentes substratos apresentaram diferença significativa, sendo o biofertilizante de caranguejo o melhor substrato entre os apresentados (Tabela 3), com valor médio de $8,58 \mathrm{~g}$, seguido pelo substrato de fermentação aeróbio (4,78 g) e os outros dois substratos (menos que $1,8 \mathrm{~g}$ ).

Tabela 3. Valores médios de matéria seca total de amendoim cultivado em diferentes substratos.

\begin{tabular}{cccc}
\hline \multicolumn{4}{c}{ MST } \\
\hline \multicolumn{4}{c}{ Substratos } \\
\hline Areia + arisco & Caranguejo & Anaeróbio & Aeróbio \\
$1,8 \mathrm{c}$ & $8,58 \mathrm{a}$ & $1,63 \mathrm{c}$ & $4,78 \mathrm{~b}$ \\
\hline MG & 4,2 & $\mathrm{CV}$ & 35 \\
DMS & 2,49 & & \\
\hline
\end{tabular}

Médias seguidas da mesma letra minúscula não diferem estatisticamente entre si (Tukey 0,05); DMS=diferença mínima significativa; $\mathrm{CV}=$ coeficiente de variação; $\mathrm{MG}=$ média geral.

Resultado semelhante foi obtido por Sousa et al. (2013), que, estudando amendoim irrigado com água salina em diferentes substratos, observou que o biofertilizante de caranguejo apresentou os maiores valores neste quesito do que o biofertilizante bovino e no substrato sem biofertilizante.

O maior valor de matéria seca total sob diferentes níveis de salinidade foi obtido na água com $\mathrm{CE} \quad 0,8 \mathrm{dS} \quad \mathrm{m}^{-1}$ (Tabela 4), demonstrando que a salinidade da água afeta diretamente o crescimento da cultura do amendoim. Correia (2005) também encontrou resultado similar, no seu estudo com diversos níveis de salinidade na cultura do amendoim, em que os menores valores de $\mathrm{CE}$ apresentaram 
valores de matéria seca total superiores aos submetidos à alta salinidade.

Tabela 4. Valores médios de matéria seca total de amendoim sob diferentes níveis de água salina.

\begin{tabular}{|c|c|}
\hline Água de Irrigação & MST \\
\hline $0,8 \mathrm{dS} \mathrm{m}^{-1}$ & $5,19 \mathrm{a}$ \\
\hline $4 \mathrm{dS} \mathrm{m}^{-1}$ & $3,2 \mathrm{~b}$ \\
\hline DMS & 1,3 \\
\hline $\mathrm{CV}$ & 35 \\
\hline $\begin{array}{l}{ }^{1}=\text { Testemunha; Médias } \\
\text { minúscula na linha não d } \\
\text { (Tukey 0,05); DMS=di } \\
\mathrm{CV}=\text { coeficiente de variaçâ }\end{array}$ & $\begin{array}{l}\text { seguidas da mesma letra } \\
\text { diferem estatisticamente entre si } \\
\text { liferença mínima significativa; } \\
\text { ção. }\end{array}$ \\
\hline
\end{tabular}

Na Tabela 5 tem-se o teste de comparação de médias da área foliar das plantas de amendoim analisadas, pode-se verificar que apenas o substrato adubado com biofertilizante bovino de fermentação aeróbia apresentou diferença significativa com o aumento da condutividade elétrica (CE) da água de irrigação de 0,8 para 4,0 $\mathrm{dSm}^{-1}$. Quando aumentou a CE, a área foliar foi reduzida em cerca de 53,55\%. Verifica-se ainda que houve diferença significativa entre os substratos utilizados.

Gomes et al. (2011) estudando plantas de milho irrigadas com alta e baixa salinidade sob diferentes espaçamentos, também observaram um decréscimo nos valores de área foliar com o aumento da salinidade da água de irrigação. A redução no crescimento foliar representa um mecanismo de defesa das plantas sob condições de estresse hídrico e salino, reduzindo as perdas de água por transpiração (TAIZ \& ZEIGER, 2010). Segundo Prisco e Gomes Filho (2010) pode-se afirmar que a salinidade inicialmente altera a absorção de água, nutrientes e a permeabilidade das membranas, causando desequilíbrio no balanço hídrico e nutricional das plantas. Todas essas mudanças comprometem a expansão e divisão celular, afetando o crescimento vegetativo e reprodutivo e acelerando a senescência das folhas.

$\mathrm{Na}$ Tabela 5 verifica-se que não houve diferença significativa no número de folhas para os substratos areia+arisco ${ }^{1}$, caranguejo e biofertilizante de fermentação anaeróbia quando se elevou a condutividade elétrica da água de

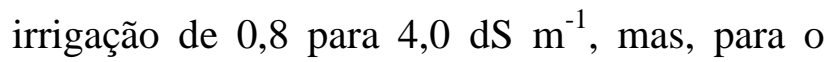
biofertilizante de fermentação aeróbia houve diferença significativa. Nesta tabela pode-se observar que para uma mesma condutividade elétrica da água de irrigação houve diferença significativa entre os substratos utilizados, sendo que os menores valores foram verificados quando se utilizou areia+arisco ${ }^{1}$ e biofertilizante de caranguejo, e os maiores valores foram verificados quando se utilizou substrato com biofertilizante bovino de fermentação anaeróbia e aeróbia.

Tabela 5. Teste de comparação de médias para a área foliar e o número de folhas da cultura do amendoim submetido a diferentes substratos e águas de irrigação

\begin{tabular}{|c|c|c|c|c|}
\hline \multirow{3}{*}{ Substratos } & \multicolumn{2}{|c|}{ Área Foliar } & \multicolumn{2}{|c|}{ Número de Folhas } \\
\hline & \multicolumn{4}{|c|}{ Água de Irrigação } \\
\hline & $0,8 \mathrm{dS} \mathrm{m}^{-1}$ & $4,0 \mathrm{dS} \mathrm{m}^{-1}$ & $0,8 \mathrm{dS} \mathrm{m}^{-1}$ & $4,0 \mathrm{dS} \mathrm{m}^{-1}$ \\
\hline Areia+arisco ${ }^{1}$ & $105,69 \mathrm{cA}$ & $70,34 \mathrm{bA}$ & $10,13 \mathrm{bA}$ & $6 \mathrm{bA}$ \\
\hline Caranguejo & $71,87 \mathrm{cA}$ & $71,4 \mathrm{bA}$ & $6,66 \mathrm{bA}$ & $8,33 \mathrm{bA}$ \\
\hline Anaeróbio & $358,37 \mathrm{bA}$ & $311,61 \mathrm{aA}$ & $31,33 \mathrm{aA}$ & $27,33 \mathrm{aA}$ \\
\hline \multirow[t]{5}{*}{ Aeróbio } & $570,38 \mathrm{aA}$ & $264,92 \mathrm{aB}$ & $34,33 \mathrm{aA}$ & $22,66 \mathrm{aB}$ \\
\hline & MG & 228,1 & MG & 18,37 \\
\hline & $\mathrm{CV}$ & 17,94 & $\mathrm{CV}$ & 19,75 \\
\hline & $\begin{array}{l}\text { DMS } \\
\text { coluna }\end{array}$ & 95,66 & $\begin{array}{l}\text { DMS } \\
\text { linha }\end{array}$ & 6,28 \\
\hline & $\begin{array}{c}\text { DMS } \\
\text { coluna }\end{array}$ & 95,66 & $\begin{array}{c}\text { DMS } \\
\text { coluna }\end{array}$ & 8,48 \\
\hline \multicolumn{5}{|c|}{$\begin{array}{l}\text { - testemunha; Médias seguidas da mesma letra maiúscula } \\
\text { na linha e minúscula na coluna não diferem } \\
\text { estatisticamente entre si (Tukey } 0,05 \text { ); DMS=diferença } \\
\text { mínima significativa; } \mathrm{CV}=\text { coeficiente de variação; } \mathrm{MG}= \\
\text { média geral }\end{array}$} \\
\hline
\end{tabular}


$\mathrm{Na}$ avaliação de plantas de amendoim submetidas a diferentes condutividades elétricas da água de irrigação e diferentes biofertilizantes Sousa et al. (2012a) verificaram que para os parâmetros de crescimento (número de folhas, altura de plantas, diâmetro do caule e área foliar), as plantas desenvolvidas na presença do biofertilizante bovino de fermentação anaeróbia apresentaram os maiores valores em comparação aos demais. Tendência similar foi evidenciado por Cavalcante et al. (2011) em plantas de pinhão-manso na presença e ausência de biofertilizante bovino.

No teste de comparação de médias para a altura das plantas (Tabela 6) verifica-se que houve diferença significativa entre os substratos utilizados, sendo que os maiores valores de altura foram observados nas plantas cultivadas em solo com biofertilizante bovino de fermentação anaeróbia e os menores valores foram observados em plantas cultivadas em substrato com biofertilizante de caranguejo.

Tabela 6. Teste de comparação de médias para a altura de plantas da cultura do amendoim submetido a diferentes substratos.

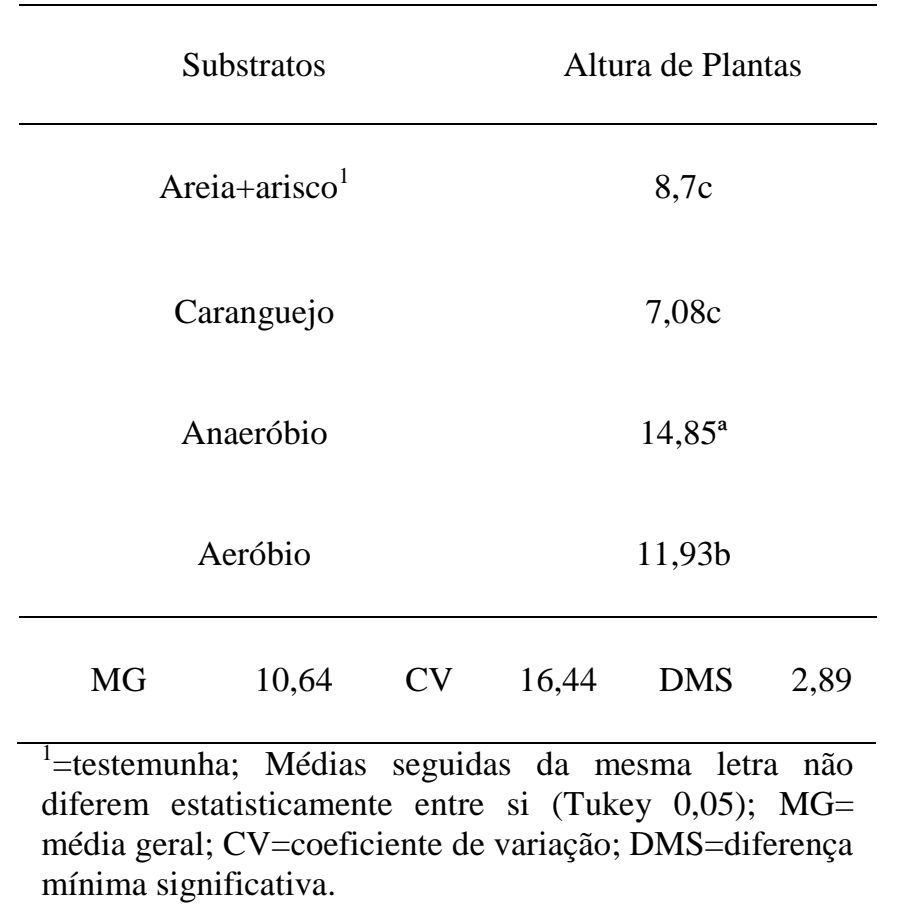

Sousa et al. (2012b) avaliando o desenvolvimento de plantas de milho sob diferentes doses de biofertilizante bovino com diferentes condutividades elétricas da água de irrigação também verificaram que na presença do biofertilizante bovino de fermentação anaeróbia os valores de altura de plantas apresentaram melhor desempenho. De forma similar, Cavalcante et al. (2010) também constataram efeito positivo do biofertilizante bovino na altura de plantas da goiaba.

Com relação à condutividade elétrica da água de irrigação utilizada, verificou-se que a maior condutividade $\left(4,0 \mathrm{dS} \mathrm{m}^{-1}\right)$ levou ao decréscimo nos valores de altura de plantas, sendo que essa diferença foi de aproximadamente $28,25 \%$.

Comportamento semelhante pôde ser observado por Gomes et al. (2011), que estudando plantas de milho irrigadas com alta e baixa salinidade sob diferentes espaçamentos, observaram um decréscimo dos valores de altura das plantas com o aumento da salinidade da água de irrigação (Tabela 7).

Tabela 7. Teste de comparação de médias para a altura de plantas da cultura do amendoim submetido a dois níveis de salinidade da água de irrigação.

$$
\text { Água de Irrigação Altura de Plantas }
$$

$$
0,8 \mathrm{dS} \mathrm{m}^{-1} \quad 12,39^{\mathrm{a}}
$$

\begin{tabular}{|c|c|c|}
\hline DMS & 1,51 & 18,66 \\
\hline \multicolumn{3}{|c|}{$\begin{array}{l}\text { Médias seguidas da mesma letra na coluna não diferem } \\
\text { estatisticamente entre si (Tukey } 0,05 \text { ); } \mathrm{DMS}=\text { diferença } \\
\text { mínima significativa; } \mathrm{CV}=\text { coeficiente de variação; } \mathrm{MG}= \\
\text { média geral. }\end{array}$} \\
\hline
\end{tabular}

$$
4,0 \mathrm{dS} \mathrm{m}^{-1} \quad 8,89 \mathrm{~b}
$$


A salinidade excessiva reduz o crescimento da planta, pois causa aumento no dispêndio de energia para absorver água do solo e realizar os ajustes bioquímicos necessários a sua sobrevivência em condições de estresse. Esta energia é desviada dos processos que conduzem ao crescimento e à produção (RHOADES et al., 2000).

\section{CONCLUSÕES}

A produção de matéria seca total do amendoim é afetada pelo aumento da salinidade da água de irrigação.

O substrato contendo biofertilizante bovino de fermentação aeróbia foi mais eficiente sobre a área foliar e o número de folhas sob irrigação com água de baixa e alta salinidade.

O substrato contendo biofertilizante bovino de fermentação anaeróbia exerce efeito atenuador para a altura de plantas irrigadas com água de baixa e alta salinidade.

\section{REFERÊNCIAS BIBLIOGRÁFICAS}

BAGHALIAN, K.; HAGHIRY, A.; NAGHAVI, M. R.; MOHAMMADI, A. Effect of saline irrigation water on agronomical and phytochemical characters of chamomile (Matricaria recutita L.). Scientia Horticulturae, v.116, n. 4, p.437-441, 2008.

BERNARDO, S.; MANTOVANI, E. C.; SOARES, A. A. Manual de Irrigação. Viçosa, UFV, 2008. 611p.

CALVET, A. S. F.; PINTO, C. M.; LIMA, R. E. M.; MAIA- JOCA, R. P. M.; BEZERRA, M. A. Crescimento e acumulação de solutos em feijão- de-corda Irrigado com águas de salinidade crescente em diferentes fases de desenvolvimento. Irriga, v. 18, n. 1, p. 148-159, 2013.

CAVALCANTE, L. F.; VIEIRA, M. SILVA; SANTOS, A. F.; OLIVEIRA, W. M.; NASCIMENTO, J. A. M. Água salina e esterco bovino líquido na formação de mudas de goiabeira cultivar paluma. Revista Brasileira de Fruticultura, v.32, n.1, p. 251-261, 2010.

CAVALCANTE, L. F.; REBEQUI, A. M.; SENA, G. S. A.; NUNES, J. C.; Irrigação com águas salinas e uso de biofertilizante bovino na formação de mudas de pinhão-manso. Irriga, v. 16, n. 3, p. 288-300, 2011.

CONAB - Companhia Nacional de Abastecimento. Boletim de Grãos. Novembro de 2013.2 In: <http://www.conab.gov.br/OlalaCMS/uploads/a rquivos/13_11_08_09_29_24_boletim_gra os_novembro_2013.pdf> Acesso em 13 de Janeiro de 2014, p. 66.

\section{CORREIA, K. B. Índices fenológicos e}

fisiológicos de amendoim sob estresse salino. Campina Grande, 2005. 67 p. Dissertação (Mestrado em Engenharia Agrícola) Universidade Federal de Campina Grande.

GARCIA, G. O.; FERREIRA, P. A.; MIRANDA, G. V.; NEVES, J. C. L.; MORAES, W. B.; SANTOS, D. B. Teores foliares dos macronutrientes catiônicos e suas relações com sódio em plantas de milho sob estresse salino. Idesia, v.25, n. 3, p.93-106, 2007.

GOMES, K. R.; AMORIM, A. V.; FERREIRA, F.J.; FILHO, F. L.; LACERDA, C. F.; 
GOMES-FILHO, E. Respostas de crescimento e fisiologia do milho submetido a estresse salino com diferentes espaçamentos de cultivo. Revista Brasileira de Engenharia Agrícola e Ambiental, v.15, n.4, p.365-370, 2011.

MEDEIROS, J. F. Qualidade da água de irrigação utilizada nas propriedades assistidas pelo "GAT" nos Estados do RN, PB, CE e avaliação da salinidade dos solos. Campina Grande: UFPB, 1992. 173 p. Dissertação Mestrado.

MUNNS, R.; TESTER, M. Mechanisms of salinity tolerance. Annual Review Plant Biology, v.59, p.651-681, 2008. <http:// dx.doi.org/10.1146/annurev.arplant.59.032607.0 92911>. 30 Sep. 2012.

PENTEADO, S. R. Adubação Orgânica: Compostos orgânicos e biofertilizantes. 2. ed. Campinas: Edição do autor, 2007.162 p.

PRISCO, J. T.; GOMES FILHO, E. Fisiologia e bioquímica do estresse salino em plantas. In: GHEYI, Hans Raj; DIAS, Nildo da Silva; LACERDA, Claudivan Feitosa de (Comp.). Manejo da salinidade na agricultura: Estudos básicos e aplicados. Fortaleza: INCT Sal, 2010. Cap. 10, p. 143-159.

RHOADES, J. D.; KANDIAH, A.; MASHALI, A. M. Uso de águas salinas para produção agrícola. Trad. GHEYI, H. R.; SOUSA, J. R. de.; QUEIROZ, J. E. Campina Grande: UFPB, 2000. 117p.

SANTOS, E. O.; SOUSA, G. G.; SILVA, G. L.; RODRIGUES, A. A. J.; VIANA, T. V. A. Irrigação com água salina na cultura do milho em solo com biofertilizante de caranguejo. Cadernos de Agroecologia, v. 8, n. 2, 2013.

SILVA, F. L. B.; LACERDA, C. F.; SOUSA, G. G.; NEVES, A. L. R.; SILVA, G. L.; SOUSA, C. H. C. Interação entre salinidade e biofertilizante bovino na cultura do feijão-decorda. Revista Brasileira de Engenharia Agrícola e Ambiental, v. 15, n. 4, p.383-389, 2011.

SILVA, M. T.; AMARAL, J. A. B. Zoneamento risco climático para a cultura do amendoim no estado do Rio Grande do Norte. Revista de Biologia e Ciência da Terra, v.7, n.2, p.93-99, 2007.

SOUSA, G. G.; SANTOS, E. O.; SILVA, G. L.; RODRIGUES, A. A. J.; VIANA, T. V. A. Irrigação do feijão-de-corda com água salina em solo com biofertilizantes. Cadernos de Agroecologia, v. 8, n. 2, p. 1-5, 2013.

SOUSA, G. G.; AZEVEDO, B. M. de; ALBUQUERQUE, A. H. P.; MESQUITA, J. B. R.; VIANA, T. V. A. Características agronômicas do amendoinzeiro sob irrigação com águas salinas em solo com biofertilizantes. Revista Agro@mbiente, v. 6, n. 2, p. 124-132, 2012a.

SOUSA, G. G.; MARINHO, A. B.; ALBUQUERQUE, A. H. P.; VIANA, T. V. A.; AZEVEDO, B. M. de. Crescimento inicial do milho sob diferentes concentrações de biofertilizante bovino irrigado com águas salinas. Revista Ciência Agronômica, Fortaleza, CE, v. 43, n. 2, p. 237-245, 2012.b

SOUSA, G. G.; LACERDA, C. F.; CAVALCANTE, L. F.; GUIMARÃES, F. V. 
A.; BEZERRA, M. E. J.; SILVA, G. L. Nutrição mineral e extração de nutrientes de planta de milho irrigada com água salina. Revista Brasileira de Engenharia Agrícola e Ambiental, v.14, n. 11, p.1143-1151, 2010.

TAIZ, L.; ZEIGER, E. Plant Physiology. 5. ed.
Sunderland: Sinauer Associates, Inc., 2010. 782 p. USDA - United States Department of Agriculture. Foreign Agricultural Service. Crop Production.

In: <http://usda01.library.cornell.edu/usda/current/ CropProd/CropProd-08-12-2013.pdf> Acesso em 13 de Janeiro de 2014, p. 45. 